\title{
ON THE CLOSURE OF THE TAME AUTOMORPHISM GROUP OF AFFINE THREE-SPACE
}

\author{
ÉRIC EDO AND PIERRE-MARIE POLONI
}

\begin{abstract}
We provide explicit families of tame automorphisms of the complex affine three-space which degenerate to wild automorphisms. This shows that the tame subgroup of the group of polynomial automorphisms of $\mathbb{C}^{3}$ is not closed, when the latter is seen as an infinite dimensional algebraic group.
\end{abstract}

\section{INTRODUCTION}

In 1965 [Sha66], Shafarevich introduced the notions of infinite-dimensional varieties and infinite-dimensional algebraic groups, now usually called indvarieties and ind-groups. His main motivation was to study the group $\mathrm{GA}_{n}(\mathbb{C})$ of polynomial automorphisms of complex affine $n$-spaces $\mathbb{A}_{\mathbb{C}}^{n}=\mathbb{C}^{n}$, which he endowed with an ind-group structure. This new approach was fruitful, since it allows him to state many nice (and tempting) results in [Sha66, Sha81, Sha95. In the present paper, we are interested in one that claims that the tame automorphisms form a dense subgroup $\mathrm{TA}_{n}(\mathbb{C})$ of $\mathrm{GA}_{n}(\mathbb{C})$. Unfortunately, Shafarevich's proof is based on another result namely that a closed subgroup $H$ of a connected ind-group $G$ with the same Lie algebra as $G$ is equal to $G$-, which turns out to be false, since Furter and Kraft constructed recently a counterexample to that statement in [FK14]. Therefore, we must reconsider the question of the density of the tame subgroup and ask it again.

Question. Is $\mathrm{TA}_{n}(\mathbb{C})$ dense in $\mathrm{GA}_{n}(\mathbb{C})$ in the topology of ind-varieties (for $n \geq 3)$ ?

Moreover, Furter and Kraft also establish the following surprising result: the subgroup $\mathrm{TA}_{2}(\mathbb{C}[z])$ of tame automorphisms of $\mathbb{C}^{3}$ that fix the last coordinate is a closed subgroup of the group $\mathrm{GA}_{2}(\mathbb{C}[z])$ of polynomial automorphisms of $\mathbb{C}^{3}$ that fix the last coordinate. In light of this, and since there were no known examples of non-tame automorphism that belong to the closure of the tame subgroup, we may even ask if the tame subgroup is closed in $\mathrm{GA}_{n}(\mathbb{C})$.

2010 Mathematics Subject Classification. 14R10.

Key words and phrases. polynomial automorphisms, tame and wild automorphisms, ind-varieties.

This research was supported in part by the ANR Grant BirPol ANR-11-JS01-004-01. 
Question. Is $\mathrm{TA}_{n}(\mathbb{C})$ closed in $\mathrm{GA}_{n}(\mathbb{C})$ in the topology of ind-varieties (for $n \geq 3)$ ?

We will show that it is not the case, when $n=3$ of course, since it is the only case where the existence of non-tame automorphisms is proved. We indeed construct families of tame automorphisms of $\mathbb{C}^{3}$ of bounded degrees which degenerate to wild (i.e. non-tame) automorphisms. In particular, we will prove that the automorphism $\varphi$ of $\mathbb{C}^{3}$ defined by

$$
\varphi=\left(x+\frac{3}{4} z^{2} y\left(\frac{3}{2} y^{2}-4 x z\right)+\frac{3}{8} z^{5}\left(\frac{3}{2} y^{2}-4 x z\right)^{2}, y+z^{3}\left(\frac{3}{2} y^{2}-4 x z\right), z\right)
$$

is not tame but is in the closure of the tame subgroup of $\mathbb{C}^{3}$. More precisely, $\varphi$ is the limit, when $t \rightarrow 0$, of the tame automorphism $\sigma_{t}$ of $\mathbb{A}_{\mathbb{C}(t)}^{3}$, which is given by

$\sigma_{t}=\left(x-\frac{3 y z}{2 t}+\frac{z^{3}}{2 t^{2}}, y-\frac{z^{2}}{t}, z\right) \circ\left(x, y, z+t^{3} x^{2}-t^{2} y^{3}\right) \circ\left(x+\frac{3 y z}{2 t}+\frac{z^{3}}{t^{2}}, y+\frac{z^{2}}{t}, z\right)$

and which has all its coefficient in $\mathbb{C}[t]$.

This example illustrates a new phenomenon concerning the length of tame automorphisms. Recall that the length of a (tame) automorphism $\sigma$ of $\mathbb{C}^{n}$ is the minimum number of triangular automorphisms that occur in a writing of $\sigma$ as composition of affine and triangular automorphisms. Recall also that Furter proved in Fur02 that the length of automorphisms of the affine plane is lower semicontinuous. That means that an automorphism of $\mathbb{C}^{2}$ of length $l$ can not be obtained as the limit of automorphisms of length $<l$. In contrast, in the above example, $\varphi$ is an automorphism of $\mathbb{C}^{3}$ of infinite length (because it is non-tame), which is the limit of the family $\sigma_{t}$ of automorphisms of length 3 .

The article is organized as follow. We fix some notations and recall the definition of tame automorphisms in Section 2. In Section 3, we recall why the group $\mathrm{GA}_{n}(\mathbb{C})$ of polynomial automorphisms of $\mathbb{C}^{n}$ has the structure of an infinite-dimensional affine algebraic variety and study the subset of $\mathrm{GA}_{3}(\mathbb{C})$ which consists of all "limits" of tame automorphisms. In particular, we show that the set of tame automorphisms of $\mathbb{C}^{3}$ of degree at most $d$ is a constructible set in $\mathrm{GA}_{3}(\mathbb{C})$ for all $d \geq 1$. Finally, we give concrete examples of non-tame automorphisms which belong to the closure of the tame subgroup in Section 4.

\section{TAME AUTOMORPHISMS}

Let $n \geq 1$ be an integer and $R$ be a commutative algebra over a field $k$. We denote by $R^{[n]}$ the polynomial algebra in $n$ variables over $R$. A polynomial map of $\mathbb{A}_{R}^{n}=\mathbb{A}_{k}^{n} \times_{\operatorname{Spec}(k)} \operatorname{Spec}(R)$ is a map $f$ from $\mathbb{A}_{R}^{n}$ to itself of the form

$$
f:\left(x_{1}, \ldots, x_{n}\right) \mapsto\left(f_{1}\left(x_{1}, \ldots, x_{n}\right), \ldots, f_{n}\left(x_{1}, \ldots, x_{n}\right)\right)
$$


where the $f_{i}$ 's belong to the polynomial ring $R\left[x_{1}, \ldots, x_{n}\right]$. We will denote by $f=\left(f_{1}, \ldots, f_{n}\right)$ such a map and we define its degree by

$$
\operatorname{deg}(f)=\max \left\{\operatorname{deg}\left(f_{i}\right) \mid i=1, \ldots, n\right\} .
$$

We will also denote by $f^{*}$ the corresponding $R$-algebra endomorphism of $R\left[x_{1}, \ldots, x_{n}\right]$, which is given by $f^{*}\left(P\left(x_{1} \ldots, x_{n}\right)\right)=P\left(f_{1}, \ldots, f_{n}\right)$ for every element $P \in R\left[x_{1}, \ldots, x_{n}\right]$. Notice in particular that $f^{*}\left(x_{i}\right)=f_{i}$ for all $i$.

The composition of two polynomial maps $f$ and $g$ is simply defined by $g \circ f=\left(g_{1}\left(f_{1}, \ldots, f_{n}\right), \ldots, g_{n}\left(f_{1}, \ldots, f_{n}\right)\right)$. We denote by $\mathrm{GA}_{n}(R)$ the group of (algebraic) automorphisms of $\mathbb{A}_{R}^{n}$ over $\operatorname{Spec}(R)$. An element $f \in \mathrm{GA}_{n}(R)$ is simply an invertible polynomial map from $\mathbb{A}_{R}^{n}$ to $\mathbb{A}_{R}^{n}$ whose inverse $f^{-1}$ is also a polynomial map. We denote by

$$
\operatorname{Aff}_{n}(R)=\left\{f \in \mathrm{GA}_{n}(R) \mid \operatorname{deg}(f)=1\right\}
$$

the affine subgroup of $\mathrm{GA}_{n}(R)$ and by

$$
\mathrm{BA}_{n}(R)=\left\{\left(f_{1}, \ldots, f_{n}\right) \in \mathrm{GA}_{n}(R) \mid f_{i} \in R\left[x_{i}, \ldots, x_{n}\right] \text { for all } i=1 \ldots n\right\}
$$

the subgroup of triangular automorphisms.

The subgroup of tame automorphisms of $\mathbb{A}_{R}^{n}$ is denoted by $\mathrm{TA}_{n}(R)$. It is the subgroup of $\mathrm{GA}_{n}(R)$ generated by $\operatorname{Aff}_{n}(R)$ and $\mathrm{BA}_{n}(R)$. An element of $\mathrm{GA}_{n}(R) \backslash \mathrm{TA}_{n}(R)$ is called wild. The Tame Generators Problem asks for the existence of such automorphisms in the case where $R=k$ is a field.

Question 2.1 (Tame Generators Problem). Does it hold that $\mathrm{GA}_{n}(k)=$ $\mathrm{TA}_{n}(k)$ ?

When $n=1$, the answer is trivially yes. When $n=2$, the answer is also positive by the famous Jung-van der Kulk's theorem (cf. [Jun42, vdK53]), which asserts moreover that, for any field $k$, the group $\mathrm{GA}_{2}(k)=\mathrm{TA}_{2}(k)$ is the amalgamated free product of $\mathrm{Aff}_{2}(k)$ and $\mathrm{BA}_{2}(k)$ along their intersection.

Note that we can consider $\mathrm{GA}_{2}(k[z])$ as a subgroup of $\mathrm{GA}_{3}(k)$ via the map sending an element $\left(f_{1}, f_{2}\right) \in \mathrm{GA}_{2}(k[z])$ onto the corresponding automorphism $\left(f_{1}, f_{2}, z\right)$ in $\mathrm{GA}_{3}(k)$, which fixes the last coordinate. Then, Shestakov and Umirbaev [SU04,SU04b] proved the following impressive result.

Theorem 2.2 (Shestakov, Umirbaev, 2004). Let $k$ be a field of characteristic zero. Viewing $\mathrm{GA}_{2}(k[z])$ and $\mathrm{TA}_{2}(k[z])$ as subgroups of $\mathrm{GA}_{3}(k)$, we have

$$
\mathrm{GA}_{2}(k[z]) \cap \mathrm{TA}_{3}(k)=\mathrm{TA}_{2}(k[z]) .
$$

This answers negatively the Tame Generators Problem in dimension 3 in characteristic zero. For example, the famous Nagata automorphism

$$
\left(x-2 y\left(y^{2}+z x\right)-z\left(y^{2}+z x\right)^{2}, y+z\left(y^{2}+z x\right), z\right)
$$

is a wild automorphism of $\mathbb{C}^{3}$. Indeed, the amalgamated free product structure on $\mathrm{GA}_{2}(\mathbb{C}(z))$ gives an algorithm to check if a given element in $\mathrm{GA}_{2}(\mathbb{C}[z])$ is in $\mathrm{TA}_{2}(\mathbb{C}[z])$ or not (see Fur97]), and it turns out that the first 
two components of Nagata's automorphism correspond to an automorphism in $\mathrm{GA}_{2}(\mathbb{C}[z]) \backslash \mathrm{TA}_{2}(\mathbb{C}[z])$. When $n \geq 4$, Question 2.1] is still open.

\section{Limits OF TAME AUTOMORPHISMS}

We will now recall how one can see $\mathrm{GA}_{n}(\mathbb{C})$ as an ind-variety (i.e. infinite dimensional algebraic variety) and which topology we consider on it. To this purpose, it is convenient to work with a fixed integer $n \geq 2$ (for us, it will be $n=3$ ) and to let $\mathcal{G}=\mathrm{GA}_{n}(\mathbb{C})$. Then, we consider the filtration on $\mathcal{G}$ given by the degree and we define for every $d \geq 1$ the subset

$$
\mathcal{G}_{\leq d}=\left\{f \in \mathcal{G}=\mathrm{GA}_{n}(\mathbb{C}) \mid \operatorname{deg}(f) \leq d\right\}
$$

of polynomial automorphisms of degree at most $d$. More generally, a subset $S \subset \mathcal{G}$ be given, we let

$$
S_{\leq d}:=S \cap \mathcal{G}_{\leq d}=\{f \in S \mid \operatorname{deg}(f) \leq d\} .
$$

One can show (see e.g. [Fur09]) that each $\mathcal{G}_{\leq d}$ has the structure of an affine algebraic variety and is closed in $\mathcal{G}_{\leq d+1}$ in the Zariski topology. Therefore, $\mathrm{GA}_{n}(\mathbb{C})=\mathcal{G}=\bigcup_{d \geq 1} \mathcal{G}_{\leq d}$ is an ind-variety in the sense of Shafarevich. As usual, we endow it with the ind-topology in which a subset $S \subset \mathcal{G}$ is closed if and only if every subset $S_{\leq d}$ is closed in $\mathcal{G}_{\leq d}$ in the Zariski topology.

In the present paper, we focus our attention on the subset of tame automorphisms of affine three-space, which we will denote by $\mathcal{T}$ in the sequel. We would like to investigate which automorphisms of $\mathbb{C}^{3}$ can be obtain as limits of such tame automorphisms in the following sense.

Definition 3.1. Let $S \subset \mathcal{G}$ be a subset and let $f \in \mathcal{G}$. We say that $f$ is limit of elements of $S$ if there exist a positive integer $d \geq 1$ and a subset $U \subset S_{\leq d}$ which is locally closed in $\mathcal{G}_{\leq d}$ such that $f \in \bar{U}$.

Following [FK14, we say that a subset $S \subset \mathcal{G}$ is weakly closed if $S$ contains all limits of elements of $S$.

Together with a valuative criterion due to Furter Fur09, Theorem 3.2 allows us to make this definition of limit more concrete in the case of tame automorphisms of $\mathbb{C}^{3}$ (see Corollary 3.4 below). This result follows from the theory of Shestakov and Umirbaev, which provides an algorithm to decompose every tame automorphism of $\mathbb{C}^{3}$ as a product of affine and triangular maps.

Theorem 3.2. For each $d \geq 1$, there exist positive integers $m=m(d)$ and $k=k(d)$, depending only on $d$, such that every tame automorphism $f \in \mathcal{T}_{\leq d}$ of $\mathbb{C}^{3}$ of degree at most $d$ can be written as a composition $f=f_{1} \circ \ldots \circ f_{m}$, where each $f_{i}$ is either affine, or a triangular automorphism of $\mathbb{C}^{3}$ of degree at most $k$.

Proof. The proof is based on Shestakov-Umirbaev theory of reduction of tame automorphisms of $\mathbb{C}^{3}$. These reductions involve affine and elementary automorphisms. Let us recall that an automorphism $f$ of $\mathbb{C}^{n}$ is called 
elementary automorphism if it is of the form

$$
f=\left(x_{1}, \ldots, x_{i-1}, x_{i}+P, x_{i+1}, \ldots, x_{n}\right)
$$

for some $1 \leq i \leq n$ and some polynomial $P \in \mathbb{C}\left[x_{1}, \ldots, \widehat{x_{i}}, \ldots, x_{n}\right]$ which does not depend on the variable $x_{i}$. We denote by $\mathcal{E}$ the set of elementary automorphisms of $\mathbb{C}^{3}$ and by $\mathcal{E}_{\leq d}$ the subset of those which are of degree at most $d$. Remark that a triangular automorphism of $\mathbb{C}^{3}$ of degree $d$ is equal to the composition of one affine automorphism and two elements of $\mathcal{E}_{\leq d}$.

In SU04b] Shestakov and Umirbaev did not consider the usual degree for a polynomial automorphism $f$, but the one that is given by the sum of the degrees of the components of $f$. Let us denote it by $\operatorname{sdeg}(f)$. So, we let $\operatorname{sdeg}\left(\left(f_{1}, f_{2}, f_{3}\right)\right)=\operatorname{deg}\left(f_{1}\right)+\operatorname{deg}\left(f_{2}\right)+\operatorname{deg}\left(f_{3}\right)$ for all automorphism $f=\left(f_{1}, f_{2}, f_{3}\right)$ of $\mathbb{C}^{3}$. According to the theory developed in SU04b, for every non-affine tame automorphism $f$ of $\mathbb{C}^{3}$, one of the following properties holds:

(1) $f$ admits an elementary reduction, i.e. there exists an elementary automorphism $e \in \mathcal{E}$ such that $\operatorname{sdeg}(e \circ f)<\operatorname{sdeg}(f)$.

(2) $f$ admits a reduction of Type I or II and then, there exist an elementary automorphism $e \in \mathcal{E}$ and an affine one $a \in \mathcal{G}_{\leq 1}$ such that $\operatorname{sdeg}(e \circ a \circ f)<\operatorname{sdeg}(f)$.

(3) $f$ admits a reduction of Type III and then, there exist an elementary automorphism $e \in \mathcal{E}$, an elementary automorphism $e_{2} \in \mathcal{E}_{\leq 2}$ of degree 2 and an affine automorphism $a \in \mathcal{G}_{\leq 1} \operatorname{such}$ that $\operatorname{sdeg}\left(e \circ e_{2} \circ\right.$ $a \circ f)<\operatorname{sdeg}(f)$.

Actually, Shestakov and Umirbaev also considered another kind of reduction, called of Type IV. But Kuroda proved that this one never occurs (see [Kur10]). Thus, any tame automorphism $f \in \mathcal{T}_{\leq d}$ can be reduced to an affine automorphism by using at most $3 d-3$ reductions as above. So, to conclude the proof, it only remains to show that all elementary maps that appear in a decomposition of an element of $\mathcal{T}_{\leq d}$ can be taken with degrees bounded by a number $k=k(d)$ which depends only on $d$.

Let us first consider elementary reductions. Let $f=\left(f_{1}, f_{2}, f_{3}\right) \in \mathcal{T}_{\leq d}$ and suppose that there exists a polynomial $P \in \mathbb{C}[x, y]$ such that $\operatorname{deg}\left(f_{3}-\right.$ $\left.P\left(f_{1}, f_{2}\right)\right)<\operatorname{deg}\left(f_{3}\right)$. We denote by $\bar{a}$ the homogeneous part of highest degree of a polynomial $a \in \mathbb{C}\left[x_{1}, x_{2}, x_{3}\right]$.

If $\overline{f_{1}}$ and $\overline{f_{2}}$ are algebraically independent, then the equality $\overline{f_{3}}=\overline{P\left(f_{1}, f_{2}\right)}=$ $\bar{P}\left(\overline{f_{1}}, \overline{f_{2}}\right)$ easily implies that $\operatorname{deg}(P) \leq \operatorname{deg}\left(f_{3}\right) \leq d$. So, let us assume that $\overline{f_{1}}$ and $\overline{f_{2}}$ are algebraically dependent. Now, if $\overline{f_{1}} \in \mathbb{C}\left[\overline{f_{2}}\right]$, then $\overline{f_{1}}=\lambda{\overline{f_{2}}}^{\alpha}$ for some $\lambda \in \mathbb{C}^{*}$ and $1 \leq \alpha \leq d$. In this case, instead of the elementary reduction $e \circ f$ of $f$ given by $e=\left(x_{1}, x_{2}, x_{3}-P\left(x_{1}, x_{2}\right)\right)$, we can perform another elementary reduction, namely the one given $\widetilde{e}=\left(x_{1}-\lambda x_{2}^{\alpha}, x_{2}, x_{3}\right)$.

Therefore, we can suppose that $\overline{f_{1}}$ and $\overline{f_{2}}$ are algebraically dependent and that $\overline{f_{1}} \notin \mathbb{C}\left[\overline{f_{2}}\right]$ and $\overline{f_{2}} \notin \mathbb{C}\left[\overline{f_{1}}\right]$. A pair $\left(f_{1}, f_{2}\right)$ of such polynomials is called $*$-reduced in [SU04, SU04b]. Without loss of generality, we can 
assume that $d_{1}=\operatorname{deg}\left(f_{1}\right)<d_{2}=\operatorname{deg}\left(f_{2}\right) \leq d$. Following [SU04, we also let $p=d_{1} \operatorname{gcd}\left(d_{1}, d_{2}\right)^{-1}, s=d_{2} \operatorname{gcd}\left(d_{1}, d_{2}\right)^{-1}, \operatorname{deg}_{x_{2}}\left(P\left(x_{1}, x_{2}\right)\right)=p q+r$ and $\operatorname{deg}_{x_{1}}\left(P\left(x_{1}, x_{2}\right)\right)=s q_{1}+r_{1}$, where $0 \leq r<p$ and $0 \leq r_{1}<s$. Then, Theorem 3 in [SU04] gives the following inequalities.

$$
\operatorname{deg}\left(P\left(f_{1}, f_{2}\right)\right) \geq q N+d_{2} r \geq q \quad \text { and } \quad \operatorname{deg}\left(P\left(f_{1}, f_{2}\right)\right) \geq q_{1} N+d_{1} r_{1} \geq q_{1},
$$

where $N:=d_{1} d_{2} \operatorname{gcd}\left(d_{1}, d_{2}\right)^{-1}-d_{1}-d_{2}+\operatorname{deg}\left[f_{1}, f_{2}\right] \geq 2$. Since $\operatorname{deg}\left(P\left(f_{1}, f_{2}\right)\right)=$ $\operatorname{deg}\left(f_{3}\right) \leq d$, we obtain

$$
\operatorname{deg}_{x_{2}}\left(P\left(x_{1}, x_{2}\right)\right)=p q+r \leq d_{1} d+d_{1} \leq d(d+1)
$$

and

$$
\operatorname{deg}_{x_{1}}\left(P\left(x_{1}, x_{2}\right)\right)=s q_{1}+r_{1} \leq d_{2} d+d_{2} \leq d(d+1) .
$$

Thus, $\operatorname{deg}(P) \leq 2 d(d+1)$ is bounded by a constant depending only on $d$, as desired.

Finally, it remains to consider the case where $f$ admits a reduction of Type I, II or III. Let us write $\widetilde{f}=\left(g_{1}, g_{2}, f_{3}\right):=a \circ f$ if $\mathrm{f}$ admits a reduction of Type I or II and $\tilde{f}=\left(g_{1}, g_{2}, f_{3}\right):=e_{2} \circ a \circ f$ if $\mathrm{f}$ admits a reduction of Type III. Furthermore, it follows from the precise definitions in SU04b] that $\left(g_{1}, g_{2}\right)$ is a $*$-reduced pair and that $\widetilde{f}$ admits an elementary reduction $e \circ \tilde{f} \operatorname{such}$ that $\operatorname{sdeg}(e \circ \tilde{f})<\operatorname{sdeg}(f)$. By the previous discussion, we can conclude that $\operatorname{deg}(e) \leq 4 d(2 d+1)$. This proves the theorem.

Theorem 3.2 implies the following fact.

Proposition 3.3. The set $\mathcal{T}_{\leq d}$ of tame automorphisms of $\mathbb{C}^{3}$ of degree at most $d$ is a constructible subset (i.e. a finite union of locally closed subsets) of $\mathcal{G}$ for every $d \geq 1$.

Proof. Let $d \geq 1$ be fixed, and let $m$ and $k$ be the corresponding integers given by Theorem 3.2. Denote by $\mathcal{B}_{\leq k}$ the set of triangular automorphisms of $\mathbb{C}^{3}$ of degree at most $k$. Remark that this set is closed in $\mathcal{G}_{\leq k}$ and thus in $\mathcal{G}$. Consequently, the set $S:=\operatorname{Aff}_{3}(\mathbb{C}) \cup \mathcal{B}_{\leq k}$ is closed in $\mathcal{G}$. The proposition follows then by Chevalley's theorem. Indeed, the composition-map $\gamma_{m}:\left(\mathcal{G}_{\leq k}\right)^{m} \rightarrow \mathcal{G}_{\leq k^{m}}$ defined by $\gamma_{m}\left(f_{1}, f_{2}, \ldots, f_{m}\right)=f_{1} \circ f_{2} \circ \cdots \circ f_{m}$ is a morphism of algebraic varieties. Therefore, $\gamma_{m}\left(S^{m}\right)$ is a constructible set in $\mathcal{G}_{\leq k^{m}}$. Hence, $\mathcal{T}_{\leq d}=\gamma_{m}\left(S^{m}\right) \cap \mathcal{G}_{\leq d}$ is constructible too.

As a corollary, we obtain the following description of the set $\mathcal{L}$ of all limits of tame automorphisms of $\mathbb{C}^{3}$.

Corollary 3.4. The set $\mathcal{L}:=\bigcup_{d>1} \overline{\mathcal{T}_{\leq d}}$ is weakly closed.

Furthermore, an automorphism $f \in \mathcal{G}$ belongs to $\mathcal{L}$ if and only if there exists a family $\varphi_{t}=\left(\left(\varphi_{t}\right)_{1},\left(\varphi_{t}\right)_{2},\left(\varphi_{t}\right)_{3}\right)$ indexed by $t \in \mathbb{C}$ such that

(1) $\left(\varphi_{t}\right)_{i} \in \mathbb{C}[[t]]\left[x_{1}, x_{2}, x_{3}\right]$ for all $1 \leq i \leq 3$;

(2) $\varphi_{t}$ defines a tame automorphism of $\mathbb{A}_{\mathbb{C}((t))}^{3}$;

(3) $\varphi_{0}=f$. 
Proof. By Proposition 3.3, we can write every set $\mathcal{T}_{\leq k}$ as a finite union $\mathcal{T}_{\leq k}=$ $\bigcup_{i=1}^{n(k)} U_{k, i}$ of locally closed sets. In particular, the equality $\overline{\mathcal{T}_{\leq k}}=\bigcup_{i=1}^{n(k)} \overline{U_{k, i}}$ holds for every $k \geq 1$. Now, let $U \subset \mathcal{L}_{\leq d}$ be locally closed in $\mathcal{G}_{\leq d}$. Remark that

$$
U=\bigcup_{k \geq 1} \overline{\mathcal{T}_{\leq k}} \cap U=\bigcup_{k \geq 1}\left(\bigcup_{i=1}^{n(k)} \overline{U_{k, i}} \cap U\right)=\bigcup_{k \geq 1} S_{k}
$$

where all $S_{k}:=\bigcup_{i=1}^{n(k)} \overline{U_{k, i}} \cap U$ are constructible subsets of $\mathcal{G}_{\leq d}$. This implies (see e.g. Lemma 2.5.4 in [FK14]) that there exists a $k_{0} \geq 1$ such that $U=$ $\bigcup_{k=1}^{k_{0}} S_{k}$. Thus, $U \subset \overline{\mathcal{T}_{\leq k_{0}}}$ and so $\bar{U} \subset \overline{\mathcal{T}_{\leq k_{0}}} \subset \mathcal{L}$. This proves that $\mathcal{L}$ is weakly closed.

The second assertion of the corollary is a direct application of a valuative criterion due to Furter [Fur09].

To sum up, we have the three following inclusions involving $\mathcal{T}, \mathcal{L}$ and $\mathcal{G}$.

$$
\mathcal{T} \subset \mathcal{L} \subset \overline{\mathcal{T}} \subset \mathcal{G}
$$

The next section is devoted to the proof that the first inclusion is a strict one, i.e. $\mathcal{T} \varsubsetneqq \mathcal{L}$. Since we do not know whether the two others are strict or not, we would like to ask two natural questions.

Question 3.5. Is $\mathcal{L}$ closed in $\mathcal{G}$ ? In other words, does the equality $\overline{\mathcal{T}}=\mathcal{L}$ hold?

Question 3.6. Is $\mathcal{T}$ dense in $\mathcal{G}$ ?

Note that these two questions are independent. Of course, $\mathcal{L}$ could be closed and not equal to the whole $\mathcal{G}$. But, more surprisingly, even if $\mathcal{T}$ would be dense in $\mathcal{G}$, there may be some automorphisms of $\mathbb{C}^{3}$ which do not belong to $\mathcal{L}$, i.e which we can not obtain as limits of tame automorphisms of bounded degree.

Finally, it is worth mentioning that $\mathcal{L}$ and $\overline{\mathcal{T}}$ are subgroups of $\mathcal{G}$. Indeed, let us recall that, as shown by Shafarevich in Sha81, $\mathcal{G}$ is an infinitedimensional algebraic group (ind-group for short). That means that the multiplication map $\mu: \mathcal{G} \times \mathcal{G} \rightarrow \mathcal{G}$ and the inverse map $\iota: \mathcal{G} \rightarrow \mathcal{G}$ are morphisms of ind-varieties, where a map $\varphi: X=\bigcup_{d} X_{\leq d} \rightarrow Y=\bigcup_{d} Y_{\leq d}$ between two ind-varieties is called morphism, if for any $n \geq 1$ there is an $m \geq 1$ such that $\varphi\left(X_{\leq n}\right) \subset Y_{\leq m}$ and $\left.\varphi\right|_{X_{\leq n}} \rightarrow Y_{\leq m}$ is a morphism of algebraic varieties. With exactly the same arguments as for the classical case of algebraic groups (see e.g. Section 7.4 in Hum75]), one checks that the following result remains true in the context of ind-groups.

Lemma 3.7. Let $G$ be a ind-group and let $H \subset G$ be a subgroup. Then, the closure $\bar{H}$ of $H$ is a subgroup of $G$.

Corollary 3.8. The sets $\mathcal{L}$ and $\overline{\mathcal{T}}$ are subgroups of $\mathrm{GA}_{3}(\mathbb{C})$. 
Proof. By Lemma 3.7, $\overline{\mathcal{T}}$ is a subgroup of $\mathcal{G}$. On one hand, it follows from Corollary 3.4 that $\mathcal{L}$ is closed under composition. On the other hand, by a result due to Gabber (see Corollary 1.4 in [BCW82]), we have $\iota\left(\mathcal{T}_{\leq d}\right) \subset \mathcal{T}_{\leq d^{2}}$ for all $d \geq 1$, where $\iota: \mathcal{G} \rightarrow \mathcal{G}$ denotes the inversion map. Therefore,

$\iota\left(\overline{\mathcal{T}_{\leq d}}\right) \subset \overline{\mathcal{T}_{\leq d^{2}}}$ for all $d \geq 1$. This is a consequence of the fact that the inversion map is a morphism of ind-groups and of the following elementary topological argument: if $f: X \rightarrow Y$ is a continuous map between two topological spaces and if $f(A) \subset B$ for some subsets $A \subset X$ and $B \subset Y$, then $f(\bar{A}) \subset \bar{B}$. Hence, $\mathcal{L}$ is closed under inversion.

\section{EXAMPLES OF WILD LIMITS}

Notation 4.1. Let $n, m \geq 1$ be positive integers. We let $\Delta \in \mathbb{C}[x, y, z]$ be given by $\Delta=z x+y^{m+1}$ and we consider the derivation $\delta$ of $\mathbb{C}[x, y, z]$ defined by

$$
\delta=\Delta\left(z^{n} \frac{\partial}{\partial y}-(m+1) y^{m} z^{n-1} \frac{\partial}{\partial x}\right) .
$$

Since $\Delta$ is in the kernel of the triangular derivation $z^{n} \frac{\partial}{\partial y}-(m+1) y^{m} z^{n-1} \frac{\partial}{\partial x}$, $\delta$ is a locally nilpotent derivation of $\mathbb{C}[x, y, z]$. Therefore, the exponential map associated to $\delta$ is a polynomial automorphism of $\mathbb{C}^{3}$. Actually, it is an element of $\mathrm{GA}_{2}(\mathbb{C}[z])$ and we have

$$
\varphi_{\lambda}=\exp (\lambda \delta)=\left(x-\sum_{k=1}^{m+1}\left(\begin{array}{c}
m+1 \\
k
\end{array}\right) \lambda^{k} \Delta^{k} y^{m+1-k} z^{n k-1}, y+\lambda \Delta z^{n}, z\right)
$$

for all $\lambda \in \mathbb{C}$. Moreover, it follows from the theory developed by Shestakov and Umirbaev, and improved by Kuroda, that $\varphi_{\lambda}$ is a wild automorphism of $\mathbb{C}^{3}$, if $\lambda \neq 0$ (see e.g. Theorem 2.3. in Kur11]).

We can now state the main result of our paper as follows.

Theorem 4.2. If $n=2 m+1$, then

$$
\varphi_{\lambda}=\exp (\lambda \delta)=\left(x-\sum_{k=1}^{m+1}\left(\begin{array}{c}
m+1 \\
k
\end{array}\right) \lambda^{k} \Delta^{k} y^{m+1-k} z^{n k-1}, y+\lambda \Delta z^{n}, z\right)
$$

is, for all $\lambda \neq 0$, a wild automorphism of $\mathbb{C}^{3}$, which belongs to the closure of $\mathrm{TA}_{3}(\mathbb{C})$.

As a corollary, we thus obtain the following result.

Corollary 4.3. The tame automorphism subgroup is not (weakly) closed in $\mathrm{GA}_{3}(\mathbb{C})$.

Before proving our main result, let us make two remarks. First, note that the famous Nagata automorphism

$$
N=\left(x-2 y\left(y^{2}+z x\right)-z\left(y^{2}+z x\right)^{2}, y+z\left(y^{2}+z x\right), z\right)
$$

corresponds to the case $n=m=1$ (and $\lambda=1$ ), which does not satisfy the condition $n=2 m+1$. Therefore, the following question naturally shows up. 
Question 4.4. Does the Nagata automorphism belong to the closure of the tame automorphism subgroup of $\mathbb{C}^{3}$ ?

Let us also point out that every tame automorphism of $\mathbb{C}^{3}$ can be easily obtained as a limit of wild automorphisms.

Proposition 4.5. The set $\mathrm{GA}_{3}(\mathbb{C}) \backslash \mathrm{TA}_{3}(\mathbb{C})$ of wild automorphisms of $\mathbb{C}^{3}$ is dense in $\mathrm{GA}_{3}(\mathbb{C})$.

Proof. Let $\sigma$ be a tame automorphism of $\mathbb{C}^{3}$ and let $\sigma_{t}$ be the family of automorphisms of $\mathbb{C}^{3}$ defined by $\sigma_{t}:=\varphi_{t} \circ \sigma$ for all $t \in \mathbb{C}$, where $\varphi_{t}$ is the exponential map of the locally nilpotent derivation $\delta$ described above. Since $\varphi_{0}$ is simply equal to the identity map, $\sigma_{t}$ converges to $\sigma$, when $t \rightarrow 0$. Thus, $\sigma$ is a limit of wild automorphisms.

In order to prove Theorem 4.2 , we need to introduce some other notations. For every positive integer $m \geq 1$, we let

$$
P_{m}(U)=\sum_{k=0}^{m}\left(\begin{array}{c}
m+\frac{1}{2} \\
k
\end{array}\right) U^{k} \in \mathbb{Q}[U] .
$$

Note that the polynomial $P_{m}(U)$ is equal to the formal power series of $(1+U)^{m+\frac{1}{2}}$ truncated at the order $m$. We consider also the two following triangular (tame) automorphisms of $\mathbb{A}_{\mathbb{C}(t)}^{3}$.

$F_{t}=\left(x, y, z+t^{m+1}\left(t x^{2}-y^{2 m+1}\right)\right) \quad$ and $\quad G_{t}=\left(x+\frac{z^{2 m+1}}{t^{m+1}} P_{m}\left(\frac{t y}{z^{2}}\right), y+\frac{z^{2}}{t}, z\right)$.

Remark that $G_{t}$ indeed defines an automorphism of $\mathbb{A}_{\mathbb{C}(t)}^{3}$, since

$$
\frac{z^{2 m+1}}{t^{m+1}} P_{m}\left(\frac{t y}{z^{2}}\right)=\sum_{k=0}^{m}\left(\begin{array}{c}
m+\frac{1}{2} \\
k
\end{array}\right) t^{-(m+1-k)} y^{k} z^{2 m+1-2 k}
$$

is an element of $\mathbb{C}(t)[y, z]$. Finally, we set $\sigma_{t}=G_{t}^{-1} \circ F_{t} \circ G_{t} \in \mathrm{GA}_{3}(\mathbb{C}(t))$. It turns out that the map $\sigma_{t}$ has all its coefficients in $\mathbb{C}[t]$. More precisely, we have the following result.

Theorem 4.6. Let $\sigma_{t}$ be the tame automorphism of $\mathbb{A}_{\mathbb{C}(t)}^{3}$ defined above. Then all three components of $\sigma_{t}$ are elements of $\mathbb{C}[t][x, y, z]$. Moreover, putting $t=0$ in their formulas, we get a wild polynomial automorphism of $\mathbb{C}^{3}$, whose last two components are $y-4 z^{2 m+1}\left(x z-\left(\begin{array}{c}m+\frac{1}{2} \\ m+1\end{array}\right) y^{m+1}\right)$ and $z$, respectively.

Proof. Remark that the inverse $G_{t}^{-1}$ of $G_{t}$ is given by

$$
G_{t}^{-1}=\left(x-\frac{z^{2 m+1}}{t^{m+1}} P_{m}\left(\frac{t y-z^{2}}{z^{2}}\right), y-\frac{z^{2}}{t}, z\right) .
$$

Note that $z^{2 m+1} P_{m}\left(\frac{t y-z^{2}}{z^{2}}\right) \in \mathbb{C}[t][x, y, z]$. In particular, the components of $\sigma_{t}=G_{t}^{-1} \circ F_{t} \circ G_{t}$ are all elements of $\mathbb{C}\left[t, t^{-1}\right][x, y, z]$. Let us denote them by $X:=\sigma_{t}^{*}(x), Y:=\sigma_{t}^{*}(y)$ and $Z:=\sigma_{t}^{*}(z)$, respectively. By construction, 
it is clear that $\sigma_{t}$ is a tame automorphism of $\mathbb{A}_{\mathbb{C}(t)}^{3}$. We will further prove the following assertions.

(1) $X, Y, Z \in \mathbb{C}[t, x, y, z]$.

(2) $Z \equiv z \bmod (t)$.

(3) $Y \equiv y-4 z^{2 m+1}\left(x z-\left(\begin{array}{c}m+\frac{1}{2} \\ m+1\end{array}\right) y^{m+1}\right) \bmod (t)$.

(4) $\tilde{\sigma}:=\left(\left.X\right|_{t=0},\left.Y\right|_{t=0},\left.Z\right|_{t=0}\right)$ is a wild automorphism of $\mathbb{C}^{3}$.

Let us first compute $Z$. Setting $T=\frac{t y}{z^{2}}$, we get $y+\frac{z^{2}}{t}=\frac{z^{2}}{t}(1+T)$, and thus

$$
\begin{aligned}
Z & =z+t^{m+1}\left(t\left(x+\frac{z^{2 m+1}}{t^{m+1}} P_{m}(T)\right)^{2}-\left(\frac{z^{2}}{t}(1+T)\right)^{2 m+1}\right) \\
& =z+t^{m+2} x^{2}+2 t x z^{2 m+1} P_{m}(T)+\frac{z^{4 m+2}}{t^{m}}\left(P_{m}(T)^{2}-(1+T)^{2 m+1}\right) .
\end{aligned}
$$

Since $P_{m}(U)$ is the formal power series of $(1+U)^{m+\frac{1}{2}}$ truncated at the order $m$, we have $P_{m}(U)^{2} \equiv(1+U)^{2 m+1}$ modulo $\left(U^{m+1}\right)$. So, $P_{m}(T)^{2} \equiv(1+$ $T)^{2 m+1}$ modulo $\left(t^{m+1}\right)$ and we obtain therefore that $Z=\sigma_{t}^{*}(z) \in \mathbb{C}[t, x, y, z]$ and $\left.Z\right|_{t=0}=z$. This proves Assertion (2).

Now, $Y=\sigma_{t}^{*}(y)=y+\frac{z^{2}}{t}-\frac{Z^{2}}{t}=y-(Z+z) \frac{Z-z}{t}$. Since the formal power series of $(1+U)^{m+\frac{1}{2}}$ truncated at the order $m+1$ is equal to $P_{m}(U)+$ $\left(\begin{array}{c}m+\frac{1}{2} \\ m+1\end{array}\right) U^{m+1}$, we have $P_{m}(U)^{2} \equiv(1+U)^{2 m+1}-2\left(\begin{array}{c}m+\frac{1}{2} \\ m+1\end{array}\right) U^{m+1}$. From this, we deduce that $P_{m}(T)^{2} \equiv(1+T)^{2 m+1}-2\left(\begin{array}{c}m+\frac{1}{2} \\ m+1\end{array}\right) T^{m+1}$ modulo $\left(t^{m+2}\right)$, where $T=\frac{t y}{z^{2}}$. Thus,

$$
\begin{aligned}
Z & \equiv z+2 t x z^{2 m+1} P_{m}(T)+\frac{z^{4 m+2}}{t^{m}}\left(-2\left(\begin{array}{c}
m+\frac{1}{2} \\
m+1
\end{array}\right) T^{m+1}\right) & \bmod \left(t^{2}\right) \\
& \equiv z+2 t x z^{2 m+1}-2\left(\begin{array}{c}
m+\frac{1}{2} \\
m+1
\end{array}\right) t y^{m+1} z^{2 m} & \bmod \left(t^{2}\right) .
\end{aligned}
$$

Therefore, $\sigma_{t}^{*}(y)=y-(Z+z) \frac{Z-z}{t} \in \mathbb{C}[t][x, y, z]$ and

$$
\left.Y\right|_{t=0}=y-4 z^{2 m+1}\left(x z-\left(\begin{array}{c}
m+\frac{1}{2} \\
m+1
\end{array}\right) y^{m+1}\right) .
$$

This proves Assertion (3). For $X=\sigma_{t}^{*}(x)$, we have

$$
\begin{aligned}
X & =x+\frac{z^{2 m+1}}{t^{m+1}} P_{m}\left(\frac{t y}{z^{2}}\right)-\frac{Z^{2 m+1}}{t^{m+1}} P_{m}\left(\frac{t y+z^{2}-Z^{2}}{Z^{2}}\right) \\
& =x+\frac{1}{t^{m+1}}\left(z^{2 m+1} P_{m}\left(\frac{t y}{z^{2}}\right)-Z^{2 m+1} P_{m}\left(\frac{t Y}{Z^{2}}\right)\right) \\
& =x+\frac{1}{t^{m+1}} \sum_{k=0}^{m}\left(\begin{array}{c}
m+\frac{1}{2} \\
k
\end{array}\right) t^{k}\left(y^{k} z^{2 m+1-2 k}-Y^{k} Z^{2 m+1-2 k}\right)
\end{aligned}
$$


We will prove that $z^{2 m+1} P_{m}\left(\frac{t y}{z^{2}}\right)-Z^{2 m+1} P_{m}\left(\frac{t Y}{Z^{2}}\right)$, which is an element of $\mathbb{C}[x, y, z, t]$, is congruent to 0 modulo $\left(t^{m+1}\right)$. It suffices to prove that

$$
\left(z^{2 m+1} P_{m}\left(\frac{t y}{z^{2}}\right)\right)^{2}-\left(Z^{2 m+1} P_{m}\left(\frac{t Y}{Z^{2}}\right)\right)^{2} \equiv 0 \bmod \left(t^{m+1}\right)
$$

and that

$$
z^{2 m+1} P_{m}\left(\frac{t y}{z^{2}}\right)+Z^{2 m+1} P_{m}\left(\frac{t Y}{Z^{2}}\right) \not \equiv 0 \quad \bmod (t) .
$$

The second assertion holds since $z^{2 m+1} P_{m}\left(\frac{t y}{z^{2}}\right) \equiv z^{2 m+1}$ and $Z^{2 m+1} P_{m}\left(\frac{t Y}{Z^{2}}\right) \equiv$ $Z^{2 m+1} \equiv z^{2 m+1}$ modulo $(t)$. For the first one, recall that $P_{m}(U)^{2}$ is congruent to $(1+U)^{2 m+1}$ modulo $\left(U^{m+1}\right)$. Therefore, we have the following congruences modulo $\left(t^{m+1}\right)$.

$$
\left(z^{2 m+1} P_{m}\left(\frac{t y}{z^{2}}\right)\right)^{2} \equiv\left(z^{2}\right)^{2 m+1}\left(1+\frac{t y}{z^{2}}\right)^{2 m+1} \equiv\left(z^{2}+t y\right)^{2 m+1}
$$

and

$$
\left(Z^{2 m+1} P_{m}\left(\frac{t Y}{Z^{2}}\right)\right)^{2} \equiv\left(Z^{2}+t Y\right)^{2 m+1}=\left(z^{2}+t y\right)^{2 m+1} .
$$

This implies the desired result, and so Assertion (1) follows.

It remains to show Assertion (4). To check that $\widetilde{\sigma}$ is a polynomial automorphism of $\mathbb{C}^{3}$, we can consider the natural extension of $F_{t}$ and $G_{t}$ as birational maps of $\mathbb{C}_{t, x, y, z}^{4}$ fixing the first coordinate. Note that their Jacobian are both equal to 1 . Thus, the endomorphism $\varphi$ of $\mathbb{C}_{t, x, y, z}^{4}$ defined by $\varphi=(t, X, Y, Z)$ is also of Jacobian 1 and it is a birational map. This implies (see for example Corollary 1.1.34 in vdE00]) that $\varphi$ is a polynomial automorphism of $\mathbb{C}^{4}$. In particular, $\widetilde{\sigma}$ is an automorphism of $\mathbb{C}^{3}$. By Assertion (2), $\widetilde{\sigma}$ is an element of $\mathrm{GA}_{2}(\mathbb{C}[z])$. Finally, Assertion (3) and the main result of [SU04b] allow us to conclude that $\widetilde{\sigma}$ is a wild automorphism of $\mathbb{C}^{3}$, since $\left.Y\right|_{t=0}$ is a wild coordinate of $\mathbb{C}[z][x, y]$ (see e.g. Proposition 2 in [EV01]).

Example 4.7. For $m=1$, we obtain that

$\sigma_{t}=\left(x-\frac{3 y z}{2 t}+\frac{z^{3}}{2 t^{2}}, y-\frac{z^{2}}{t}, z\right) \circ\left(x, y, z+t^{3} x^{2}-t^{2} y^{3}\right) \circ\left(x+\frac{3 y z}{2 t}+\frac{z^{3}}{t^{2}}, y+\frac{z^{2}}{t}, z\right)$

is a tame automorphism of $\mathbb{A}_{\mathbb{C}(t)}^{3}$ which has all its coefficient in $\mathbb{C}[t]$. Computing explicitly these coefficients and letting $t=0$ in the formulas, we find then the following wild automorphism of $\mathbb{C}^{3}$, which is the limit when $t \rightarrow 0$ of the family $\left(\sigma_{t}\right)_{t \neq 0}$ of tame automorphisms of $\mathbb{C}^{3}$.

$$
\begin{aligned}
\widetilde{\sigma} & =\left(x+\frac{9}{8} y^{3} z^{2}-3 x y z^{3}+\frac{27}{32} y^{4} z^{5}-\frac{9}{2} x y^{2} z^{6}+6 x^{2} z^{7}, y+\frac{3}{2} y^{2} z^{3}-4 x z^{4}, z\right) \\
& =\left(x+\frac{3}{4} z^{2} y\left(\frac{3}{2} y^{2}-4 x z\right)+\frac{3}{8} z^{5}\left(\frac{3}{2} y^{2}-4 x z\right)^{2}, y+z^{3}\left(\frac{3}{2} y^{2}-4 x z\right), z\right) .
\end{aligned}
$$

Finally, we prove Theorem 4.2 . 
Proof. (of Theorem 4.2). Let $m \geq 1$ be a fixed integer. For every $\lambda \in \mathbb{C}^{*}$, let $\Psi_{\lambda}$ be the affine automorphism of $\mathbb{C}^{3}$ defined by $\Psi_{\lambda}=(a x, b y, c z)$, where

$a, b, c \in \mathbb{C}^{*}$ are chosen such that $-\left(\begin{array}{c}m+\frac{1}{2} \\ m+1\end{array}\right) b^{m+1}=1,-4 c^{2 m+1}=\lambda b$ and $a c=1$. Then, consider the automorphism $\alpha_{t}$ of $\mathbb{A}_{\mathbb{C}(t)}^{3}$ given by $\alpha_{t}=\Psi_{\lambda}^{-1} \circ$ $\sigma_{t} \circ \Psi_{\lambda}$, where $\sigma_{t}$ denotes the automorphism defined before Theorem 4.6, By Theorem 4.6, we have that $\left(\alpha_{t}\right)_{t \in \mathbb{C}^{*}}$ is a family of tame automorphisms of $\mathbb{C}^{3}$, which converges to a wild automorphism $\alpha$ of $\mathbb{C}^{3}$, when $t \rightarrow 0$. Moreover, the two last components of $\alpha$ are equal to $y+\lambda\left(x z+y^{m+1}\right) z^{2 m+1}$ and $z$, respectively. Note that these two are also the last components of $\varphi_{\lambda}=\exp (\lambda \delta)$ in the case $n=2 m+1$. Therefore, there exists a tame polynomial automorphism $f$ of $\mathbb{C}^{3}$ of the form $f=(d x+P(y, z), y, z)$ with $d \in \mathbb{C}^{*}$ and $P(y, z) \in \mathbb{C}[y, z]$ such that $f \circ \alpha=\varphi_{\lambda}$. Thus, $\left(f \circ \alpha_{t}\right)_{t \neq 0}$ is a family of tame automorphisms, which converges to $\varphi_{\lambda}$ for $t \rightarrow 0$. This proves the theorem.

\section{AcKnowledgements}

We are very grateful to Jean-Philippe Furter and Hanspeter Kraft for sharing with us preliminary version of their upcoming paper about the geometry of the automorphism group of affine $n$-space, and for fruitful discussions, which helped us to understand some subtleties of the topology of ind-groups.

\section{REFERENCES}

[BCW82] Hyman Bass, Edwin H. Connell, and David Wright, The Jacobian conjecture: reduction of degree and formal expansion of the inverse, Bull. Amer. Math. Soc. (N.S.) 7 (1982), no. 2, 287-330.

[EV01] Eric Edo and Stéphane Vénéreau, Length 2 variables of $A[x, y]$ and transfer, Ann. Polon. Math. 76 (2001), no. 1-2, 67-76. Polynomial automorphisms and related topics (Kraków, 1999).

[vdE00] Arno van den Essen, Polynomial automorphisms and the Jacobian conjecture, Progress in Mathematics, vol. 190, Birkhäuser Verlag, Basel, 2000.

[vdK53] W. van der Kulk, On polynomial rings in two variables, Nieuw Arch. Wiskunde (3) 1 (1953), 33-41.

[Fur97] Jean-Philippe Furter, On the variety of automorphisms of the affine plane, J. Algebra 195 (1997), no. 2, 604-623.

[Fur02] - On the length of polynomial automorphisms of the affine plane, Math. Ann. 322 (2002), no. 2, 401-411.

[Fur09] _ Plane polynomial automorphisms of fixed multidegree, Math. Ann. 343 (2009), no. 4, 901-920.

[FK14] Jean-Philippe Furter and Hanspeter Kraft, On the geometry of the automorphism group of affine $n$-space, in preparation (2014).

[Hum75] James E. Humphreys, Linear algebraic groups, Springer-Verlag, New York, 1975. Graduate Texts in Mathematics, No. 21.

[Jun42] Heinrich W. E. Jung, Über ganze birationale Transformationen der Ebene, J. Reine Angew. Math. 184 (1942), 161-174.

[Kur10] Shigeru Kuroda, Shestakov-Umirbaev reductions and Nagata's conjecture on a polynomial automorphism, Tohoku Math. J. (2) 62 (2010), no. 1, 75-115. 
[Kur11] - Wildness of polynomial automorphisms in three variables, preprint arXiv:1110.1466 [math.AC] (2011).

[Sha66] Igor R. Shafarevich, On some infinite-dimensional groups, Rend. Mat. e Appl. (5) 25 (1966), no. 1-2, 208-212.

[Sha81] , On some infinite-dimensional groups. II, Izv. Akad. Nauk SSSR Ser. Mat. 45 (1981), no. 1, 214-226, 240 (Russian).

[Sha95] L Letter to the editors: "On some infinite-dimensional groups. II", Izv. Ross. Akad. Nauk Ser. Mat. 59 (1995), no. 3, 224 (Russian).

[SU04a] Ivan P. Shestakov and Ualbai U. Umirbaev, Poisson brackets and two-generated subalgebras of rings of polynomials, J. Amer. Math. Soc. 17 (2004), no. 1, 181196.

[SU04b] _ The tame and the wild automorphisms of polynomial rings in three variables, J. Amer. Math. Soc. 17 (2004), no. 1, 197-227.

Université de la Nouvelle Calédonie, Bat. S, Campus de Nouville, BP R4 98851 Nouméa CEDEX, Nouvelle Calédonie.

E-mail address: eric.edo@univ-nc.nc

Universität Basel, Mathematisches Institut, Rheinsprung 21, CH-4051 Basel, SWITZERLAND.

E-mail address: pierre-marie.poloni@unibas.ch 